\title{
Disseminated Presentation of Primary Lymphoblastic Lymphoma of the Bone in a Pediatric Patient
}

\author{
Lisa A. Cao, Alya Sheikh, Xin Qing, Samuel French, Joseph L. Lasky, \\ Eduard H. Panosyan* \\ Department of Pediatrics, Harbor-UCLA, Los Angeles, USA \\ Email: "epanosyan@labiomed.org
}

Received 5 May 2015; accepted 27 June 2015; published 30 June 2015

Copyright (C) 2015 by authors and Scientific Research Publishing Inc.

This work is licensed under the Creative Commons Attribution International License (CC BY). http://creativecommons.org/licenses/by/4.0/

(c) () Open Access

\begin{abstract}
Primary non-Hodgkin's lymphoma of the bone (PLB) is extremely rare in the pediatric population with less than 100 cases reported in the English literature. Most commonly, patients present with atraumatic bone pain and grossly normal radiographic findings. PLB is in the histopathological class of "small round cell tumors of bone", as with most common bone tumors. The diagnosis is confirmed by immunohistochemical or flow cytometry based detection of tumor-specific proteins. We present a case of stage IV PLB of B-lymphoblastic type with an excellent response to chemotherapy to increase awareness among general pediatricians and pathologists about the importance of making the correct diagnosis, given the excellent prognosis for this disease.
\end{abstract}

\section{Keywords}

Primary Lymphoma of Bone, Pediatric Musculoskeletal Tumor

\section{Introduction}

Primary non-Hodgkin's lymphoma of the bone (PLB) is uncommon in adults, and it is even rarer in the pediatric population [1]. There have been less than 100 cases reported in the English literature [2]. PLB is a unique entity in that bony lesions are the primary site of origin of non-Hodgkin's lymphoma. The initial presentation is commonly atraumatic bone pain in a child or adolescent, predominantly male, with grossly normal radiographs [3]. The bony lesions are commonly found in the axial skeleton or long bones, such as the pelvis and femur, thus can be initially misdiagnosed as classic bone tumor, such as Ewing sarcoma or osteosarcoma [4]. Although rare, it is

*Corresponding author.

How to cite this paper: Cao, L.A., Sheikh, A., Qing, X., French, S., Lasky, J.L. and Panosyan, E. (2015) Disseminated Presentation of Primary Lymphoblastic Lymphoma of the Bone in a Pediatric Patient. Journal of Cancer Therapy, 6, 536-542.

http://dx.doi.org/10.4236/jct.2015.66058 
important to include malignant bone tumors in the differential diagnoses as a general pediatrician or emergency medicine provider.

The pathological finding of PLB is "small round cell tumors of bone”. Unfortunately, this is not pathognomonic for this diagnosis or any of the other common bone tumors that are predominantly seen in children and adolescents. The differential includes the Ewing sarcoma family of tumors, mesenchymal chondrosarcoma, small cell osteosarcoma, and rhabdomyosarcoma [5]. Although PLB is rare, it is important for pathologists to include as a possible diagnosis given its marked response to chemotherapy [1]. The chemotherapy regimen for Lymphoblastic PLB is similar to that used to treat acute lymphoblastic lymphoma (ALL) [6]. The diagnosis is confirmed by immunohistochemical staining for tumor markers. The predominant histologic type of PLB is diffuse large B-cell lymphoma [4]. In the few histological cases of the lymphoblastic type, several have presented in a disseminated fashion classified as stage III PLB [1].

We present a case of stage IV PLB of lymphoblastic type with an excellent response to chemotherapy to increase awareness among the general pediatricians and pathologists about the good outcomes of correct diagnosis. Parental and patient's permission was obtained for this case report.

\section{Clinical Summary}

A 9-year-old boy presented to an outside Emergency Department with a tender mass on his right shin. At that time, the parents were reassured that the mass was most likely from overuse causing a stress fracture. The patient was advised no weight-bearing on his right leg for two weeks and prescribed an NSAID for the pain. After six weeks of full activity, the patient noticed that the mass had increased in size and the pain was worsening.

At this time, the patient presented to our hospital. Besides the chief complaint, the boy had been previously healthy with a negative review of symptoms. Patient was afebrile and mildly tachycardic on presentation; the rest of his vital signs were within normal limits. Notable on the exam is a palpable $2 \mathrm{~cm}$ deformity that is tender with no areas of fluctuance. Patient was observed walking with a limp. Initial labs revealed an elevated erythrocyte sedimentation rate (ESR), C-reactive protein (CRP) at 3.59, and lactate dehydrogenase (LDH) at 352.

$\mathrm{X}$-ray of the right tibia and fibula revealed circumferential cortical thickening and periosteal elevation of the tibia (Figure 1). MRI of the right leg showed abnormal T1 hypointensity and STIR hyperintensity in the entire length of the tibia marrow. Incidentally, there was abnormal T1 and STIR signal throughout the proximal left tibial marrow and left distal femur. A periosteal defect was seen anteromedially at the right tibial mid-shaft, correlating to the patient's mass (Figure 2). A bone scan was subsequently performed showing multiple regions of abnormal marrow uptake: entire right tibia, mid and distal left femur, left femoral head and neck, and left tibial diaphysis (Figure 3).

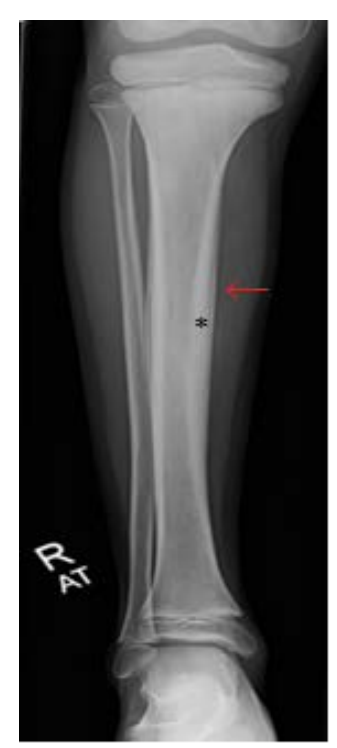

Figure 1. X-ray of tibia and fibula Periosteal elevation is denoted by the arrow and cortical thickening by the star. 


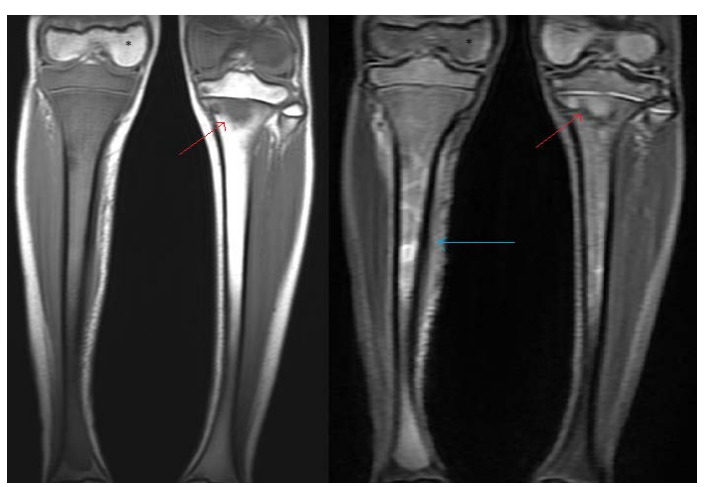

Figure 2. MRI of bilateral legs. Denoted by the blue arrow is the periosteal defect in the right tibal shaft, correlating with the patient's mass. Denoted by the red arrow is abnormal signal in the left tibia. The star marks normal bone signal in the right distal femur, as compared to the abnormal signal in the left distal femur.

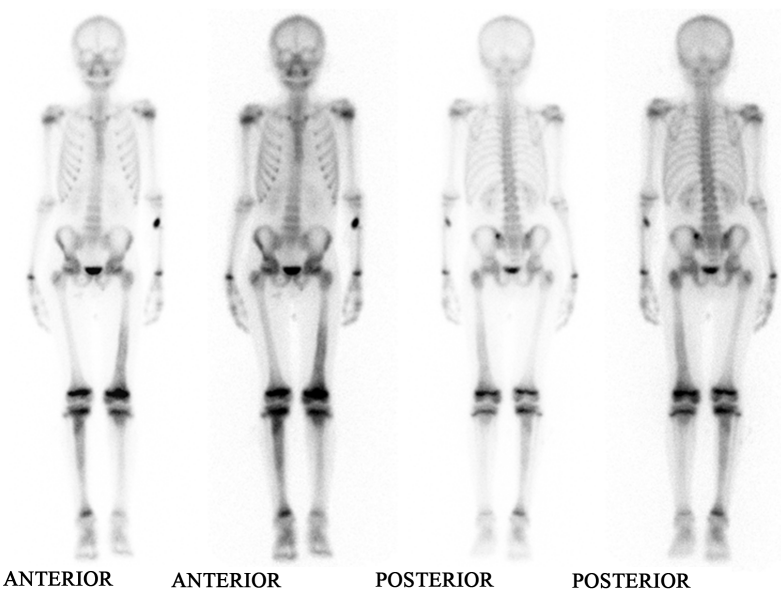

Figure 3. Bone scan demonstrating abnormal uptake in entire right tibia, mid and distal left femur, left femoral head and neck, and left tibial diaphysis.

A right proximal tibial bone biopsy was performed and showed small round blue cell tumor (Figure 4(A)). The differential diagnosis included primitive neuroectodermal tumor (PNET)/Ewing's sarcoma, lymphoma, neuroblastoma, and primary bone tumor. Unfortunately, the cytological picture could not be determined due to marked extensive crush artifact. Given that there was high suspicion for Ewing's sarcoma, a sarcoma mutation panel was ordered and was negative for translocations associated with Ewing's sarcoma, rhabdomyosarcoma, and synovial sarcoma. Immunohistochemical staining revealed the tumor cells were positive for CD10 (not shown), CD34, CD79a (not shown), PAX5, CD99 (not shown) and TdT (Figures 4(B)-(D)), and negative for CD3 and CD20 (not shown). This confirmed the diagnosis of B-lymphoblastic lymphoma of the bone. The patient's bone marrow biopsy revealed mildly increased and focal clusters of blasts that were positive for B-cell markers CD79a and PAX5 as well as TdT, suggesting minimal involvement of B-lymphoblastic lymphoma. The patient's CSF was negative for tumor cells. The patient was diagnosed with disseminated (stage IV) primary B-lymphoblastic lymphoma of the bone.

The patient was treated following the Children's Oncology Group (COG) A5971 protocol, regimen B1 for lymphoblastic lymphoma [6] [7]. After the patient completed the induction phase of the protocol, the patient was re-staged by a whole body PET/CT. It demonstrated no enlarged or hypermetabolic adenopathy. There were diffuse marrow changes involving the left femur, left greater trochanter, and left mid to distal femoral diaphysis on unenhanced CT with no corresponding abnormal FDG uptake on PET (Figure 5).

Currently, the patient is on maintenance chemotherapy. A repeat whole body PET/CT scan demonstrated no new evidence of abnormal activity to suggest disease recurrence or progression. A repeat MRI of bilateral legs showed therapeutic response to chemotherapy with decrease in size of bilateral tibial lesions (Figure 6). 


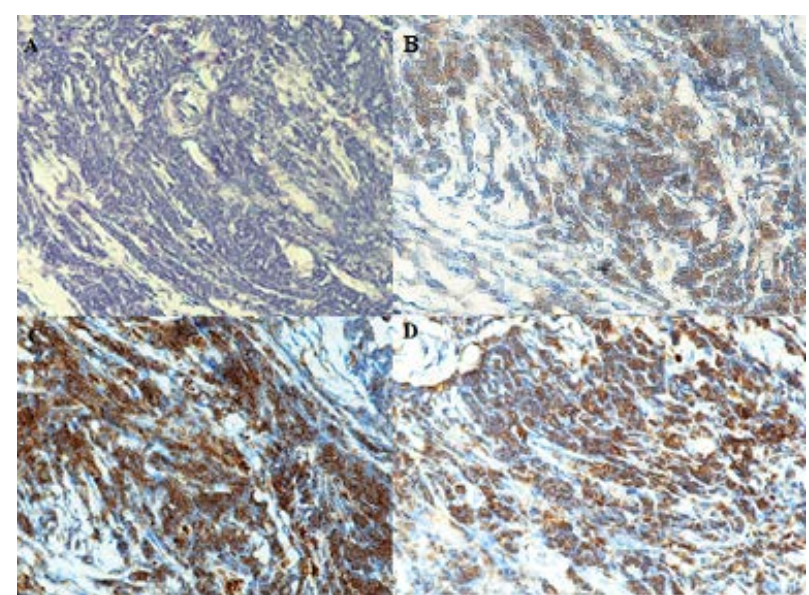

Figure 4. (A) Bone biopsy showed small round blue cell tumor with extensive crush artifact. The tumor cells are positive for PAX5 (B); CD34 (C); and TdT (D).

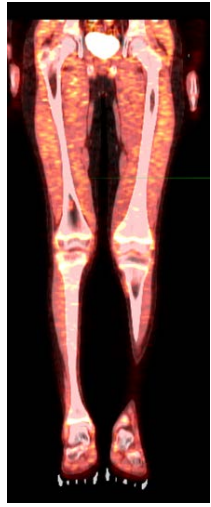

Figure 5. PET/CT scan demonstrating diffuse marrow changes in the left femur with no associated abnormal FDG uptake.

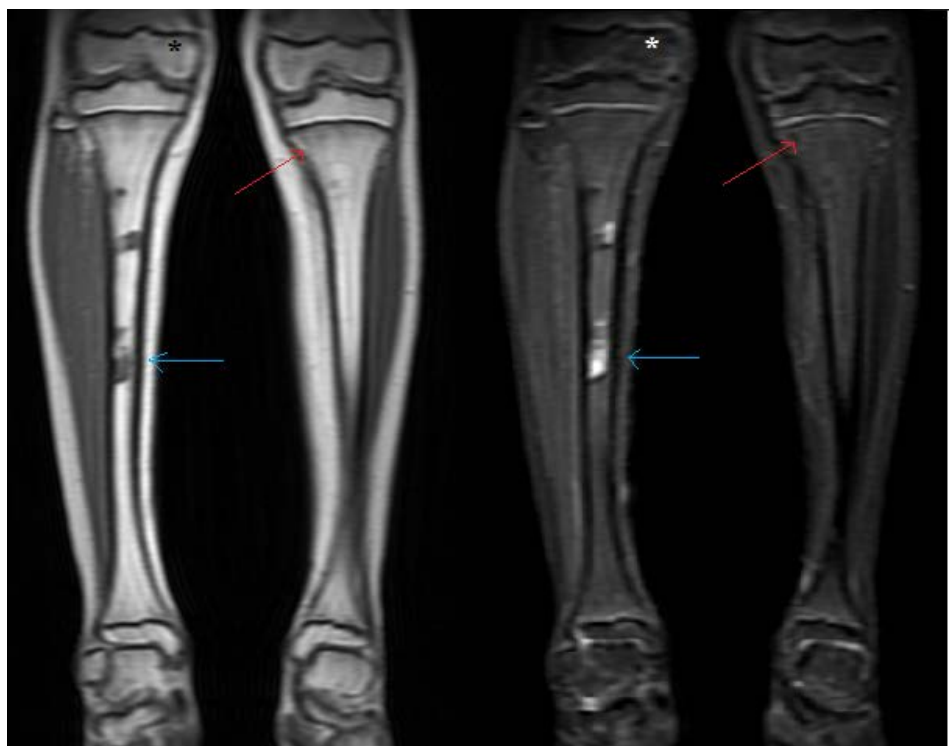

Figure 6. Repeat MRI shows persistence of the periosteal defect in the right tibial shaft, as denoted by the blue arrow. The T1 hypodensity and STIR hyperintensity in the left tibia have normalized, denoted by the red arrow. The star marks normal signal in the right distal femur. 


\section{Discussion}

PLB can present as multi-focal disease that affects multiple skeletal sites without visceral or lymph node involvement [8]. In the pediatric population, it comprises approximately $2 \%$ of all pediatric bone tumors [9]. Although extremely rare, it has been shown that PLB has had excellent outcomes with chemotherapy. Therefore, it is in the patient's best interest to confirm his or her diagnosis prior to proceeding with a treatment plan.

Prior to confirming the diagnosis of this case, the patient was thought to have metastatic Ewing's sarcoma given the preliminary pathological report of "small round blue cell tumor" and characteristic radiographic findings. If this were the case, the patient would have a poor prognosis [10]. Despite aggressive therapy, metastatic Ewing's sarcoma has a 19\% - 30\% survival rate at 3 - 5 years [3]. Fortunately, clarification of the diagnosis in our case was provided by the immunohistochemical stains. CT-guided percutaneous needle biopsy has been reported to have an overall diagnostic accuracy of $68 \%$ - $89 \%$ when used to evaluate musculoskeletal tumors [11]. However, the diagnostic accuracy of lymphoma is lower $(50 \%-67 \%)$ than other musculoskeletal tumors and has a higher re-biopsy rate [12]. Thus, in cases with a high suspicion for lymphoma, a surgical biopsy is recommended.

To successfully identify non-Hodgkin's lymphoma, immunophenotyping of the tumor cells by flow cytometry and/or immunohistochemical staining of tumor markers is required. B-cells are identified using CD19, CD20, CD79a, and PAX5, while T-cell markers are CD2, CD3, CD5, and CD7 [13]. To identify lymphoblastic lymphoma, which is the subtype that our patient had, a panel including CD3, CD20, CD34, CD43, CD45, CD79a, PAX5, CD99, and TdT can be helpful. CD43 and CD79a are useful stains for differentiating lymphoblastic lymphoma/leukemia from Ewing's sarcoma. However, CD43 and CD79a can be expressed in both T-and B-lymphoblastic lymphoma/leukemia, and therefore, are not lineage specific.

In addition to immunohistochemistry, we ruled out Ewing's sarcoma and other sarcomas in our patient using molecular genetics, which was negative for EWS-WT1, EWS-FLI1, EWS-ERG, PAX3/FKHR, PAX7/FKHR, SYT-SSX1, and SYT-SSX2 translocations. Ewing's sarcoma and PNET tumor cells commonly have chromosomal translocations that can be identified in $85 \%-95 \%$ of tumors [14]. For pathologists, CD99 is the most useful tumor marker for the diagnosis of Ewing's sarcoma. Unfortunately, it is non-specific and is highly expressed in a number of other tumors, including T- and B-lymphoblastic lymphoma and leukemia, and other sarcomas. This marker would not have been useful in our case to differentiate between Ewing's sarcoma and lymphoblastic lymphoma. That is the case for the rest of the immunomarkers used to identify Ewing's sarcoma, such as FLI-1 and vimentin [15].

PLB can present radiographically benign or near normal [16]. In our case, the abnormalities were subtle. Another reason why we thought the patient had Ewing's sarcoma was the classic radiographic signs: cortical thickening and periosteal elevation. However, these signs are not pathognomonic and can be seen in a variety of other bone pathologies [17]. MRI is sensitive and will pick up a soft tissue mass associated with PLB 84\% - 100\% of the time [18]. In our case, we discovered multiple bone lesions incidentally when performing the MRI to further define our target lesion.

Unfortunately, MRI is not a good imaging method to demonstrate treatment response. As evidenced in a case report by Marina et al, after 8 years of treatment and complete remission clinically, the MRI still demonstrated persistent unmodified bone marrow alteration and appreciable cortical involvement [19]. They showed that the 3-phase bone scan demonstrated inactive disease and clinical remission, which is a possible modality for disease progression. PET scans may be a promising option to monitor response to therapy [20]. As of right now, the only possible way to confirm complete remission is through repeat biopsy.

Our plan to follow our patient's disease progression has been through serial MRI imaging. We will repeat a PET/CT scan once he has completed his full course of chemotherapy. At that time, if we feel that the bony lesions are still prominent, we will consider repeat biopsy.

\section{Conclusion}

We present a case of stage IV primary lymphoma of the bone (PLB), lymphoblastic type with an excellent response to chemotherapy. Our hope is for general pediatricians to recognize the importance of confirming the correct diagnosis as a patient's prognosis is dependent on this and to be aware of PLB in the differential diagnosis of bone pain. In addition, pathologists play an important role in helping to make the diagnosis by performing immunohistochemical staining for tumor specific proteins and sending appropriate molecular testing. For on- 
cologists, it is important to note that there is a limited role of imaging in the evaluation of therapy responses for this disease, and that good clinical judgment plays an important role in following disease response.

\section{References}

[1] Lones, M.A., Perkins, S.L., Sposto, R., Tedeschi, N., Kadin, M.E., Kjeldsberg, C.R., Wilson, J.F., Zwick, D.L. and Cairo, M.S. (2002) Non-Hodgkin's Lymphoma Arising in Bone in Children and Adolescents Is Associated With an Excellent Outcome: A Children's Cancer Group Report. Journal of Clinical Oncology, 20, 2293-2301. http://dx.doi.org/10.1200/JCO.2002.06.017

[2] Fox, M.G., Marti, J.K., Bachmann, K.R., LeGallo, R.D. and Foster, W.C. (2015) Epiphyseal Presentation of Non-Hodgkin’s Lymphoma of Bone in Two Pediatric Patients-One with Primary Lymphoma of Bone. Skeletal Radiology, 44, 587-595. http://dx.doi.org/10.1007/s00256-014-2010-7

[3] Arndt, C.A., Rose, P.S., Folpe, A.L. and Laack, N.N. (2012) Common Musculoskeletal Tumors of Childhood and Adolescence. Mayo Clinic Proceedings, 87, 475-487. http://dx.doi.org/10.1016/j.mayocp.2012.01.015

[4] Bao, J. (2012) Young Males with Primary Lymphoma of Bone Presenting with Musculoskeletal Pain Are Prone to Be Misdiagnosed as Ankylosing Spondylitis: A Case Report. Rheumatology International, 32, 253-254. http://dx.doi.org/10.1007/s00296-010-1776-8

[5] Hameed, M. (2007) Small Round Cell Tumors of Bone. Archives of Pathology and Laboratory Medicine, 131, 192204.

[6] Termuhlen, A.M., Smith, L.M., Perkins, S.L., Lones, M., Finlay, J.L., Weinstein, H., Gross, T.G. and Abromowitch, M. (2012) Outcome of Newly Diagnosed Children and Adolescents with Localized Lymphoblastic Lymphoma Treated on Children's Oncology Group Trial A5971: A Report from the Children’s Oncology Group. Pediatric Blood \& Cancer, 59, 1229-1233. http://dx.doi.org/10.1002/pbc.24149

[7] Termuhlen, A.M., Smith, L.M., Perkins, S.L., Lones, M., Finlay, J.L., Weinstein, H., Gross, T.G. and Abromowitch, M. (2013) Disseminated Lymphoblastic Lymphoma in Children and Adolescents: Results of the COG A5971 Trial: A Report from the Children’s Oncology Group. British Journal of Haematology, 162, 792-801. http://dx.doi.org/10.1111/bjh.12460

[8] Messina, C. Ferreri, A.J., Govi, S., Bruno-Ventre, M., Gracia Medina, E.A., Porter, D., Radford, J., Heo, D.S., Park, H.Y., Pro, B., Jayamohan, J., Visco, C., Scarfo, L., Zucca, E., Gospodarowicz, M. and Christie, D., International Extranodal Lymphoma Study Group (IELSG) (2014) Clinical Features, Management and Prognosis of Multifocal Primary Bone Lymphoma: A Retrospective Study of the International Extranodal Lymphoma Study Group (the IELSG 14 study). British Journal of Haematology, 164, 834-840. http://dx.doi.org/10.1111/bjh.12714

[9] Furman, W.L., Fitch, S., Hustu, H.O., Callihan, T. and Murphy, S.B. (1989) Primary Lymphoma of Bone in Children. Journal of Clinical Oncology, 7, 1275-1280.

[10] Cotterill, S.J., Ahrens, S., Paulussen, M., Jurgens, H.F., Voute, P.A., Gadner, H. and Craft, A.W. (2000) Prognostic Factors in Ewing's Tumor of Bone: Analysis of 975 Patients from the European Intergroup Cooperative Ewing's Sarcoma Study Group. Journal of Clinical Oncology, 18, 3108-3114.

[11] Li, Y., Du, Y., Luo, T.Y., Yang, H.F., Yu, J.H., Xu, X.X., Zheng, H.J. and Li, B. (2014) Factors Influencing Diagnostic Yield of CT-Guided Percutaneous Core Needle Biopsy for Bone Lesions. Clinical Radiology, 69, e43-e47. http://dx.doi.org/10.1016/i.crad.2013.09.003

[12] Nouh, M.R. and Abu Shady, H.M. (2014) Initial CT-Guided Needle Biopsy of Extremity Skeletal Lesions: Diagnostic Performance and Experience of a Tertiary Musculoskeletal Center. European Journal of Radiology, 83, 360-365. http://dx.doi.org/10.1016/j.ejrad.2013.10.012

[13] Reiter, A. (2007) Diagnosis and Treatment of Childhood Non-Hodgkin Lymphoma. Hematology, 2007, 285-296. http://dx.doi.org/10.1182/asheducation-2007.1.285

[14] Aurias, A., Rimbaut, C., Buffe, C., Dubousset, J. and Mazabraud, A. (1983) Chromosomal Translocations in Ewing's Sarcoma. New England Journal of Medicine, 309, 496-498. http://dx.doi.org/10.1056/NEJM198308253090817

[15] Llombart-Bosch, A. and Navarro, S. (2001) Immunohistochemical Detection of EWS and FLI-1 Proteins in Ewing Sarcoma and Primitive Neuroectodermal Tumors: Comparative Analysis With CD99 (MIC-2) Expression. Applied Immunohistochemistry \& Molecular Morphology, 9, 255-260. http://dx.doi.org/10.1097/00129039-200109000-00010

[16] Krishnan, A., Shirkhoda, A., Tehranzadeh, J., Armin, A.R., Irwin, R. and Les, K. (2003) Primary Bone Lymphoma: Radiographic-MR Imaging Correlation. RadioGraphics, 23, 1371-1387. http://dx.doi.org/10.1148/rg.236025056

[17] Scully, R.E., Mark, E.J. and McNeely, B.U. (1981) Case Records of the Massachusetts General Hospital. Weekly Clinicopathological Exercises. Case 36-1981. New England Journal of Medicine, 305, 567-574.

[18] Mulligan, M.E., McRae, G.A. and Murphey, M.D. (1999) Imaging Features of Primary Lymphoma of Bone. American 
Journal of Roentgenology, 173, 1691-1697. http://dx.doi.org/10.2214/ajr.173.6.10584821

[19] Marina, V., Milena, R., Vesna, P., Sladana, P. and Vera, A. (2015) Complementary Roles of Bone Scintigraphy and MR Imaging in the Detection and Long-Term Follow-Up of Primary Non-Hodgkin's Bone Lymphoma in a Child-Case Report. Skeletal Radiology, 44, 863-868. http://dx.doi.org/10.1007/s00256-014-2067-3

[20] Spaepen, K., Stroobants, S., Dupont, P., Van Steenweghen, S., Thomas, J., Vandenberghe, P., Vanuytsel, L., Bormans, G., Balzarini, J., De Wolf-Peeters, C., Mortelmans, L. and Verhoef, G. (2001) Prognostic Value of Positron Emission Tomography (PET) with Fluorine-18 Fluorodeoxyglucose ([18F]FDG) after First-Line Chemotherapy in Non-Hodgkin’s Lymphoma: Is [18F]FDG-PET a Valid Alternative to Conventional Diagnostic Methods? Journal of Clinical Oncology, 19, 414-419. 